\title{
Identification of Sparse Damage in Steel-Frame Buildings Using Dense Seismic Array Measurements
}

\author{
FILIPPOS FILIPPITZIS, MONICA D. KOHLER and \\ THOMAS H. HEATON
}

\begin{abstract}
There is an unprecedented increase in the number of real-time measurements produced by permanent, dense accelerometer arrays in buildings, an example being the Community Seismic Network. In the present work, damage identification techniques are developed by coupling such datasets with linear and nonlinear finite-element models of buildings. Damage in steel-frame buildings is manifested in localized areas as cracks in beam-column connections or as an average stiffness reduction. High-fidelity linear or nonlinear finite-element models are developed to allow for realistic behavior, including modeling nonlinearities associated with the opening and closing of cracks. L1 regularization techniques and sparse Bayesian learning tools are further developed fully in the time domain to reduce ill-conditioning and account for the sparsity of damage. The effectiveness of the proposed methods in identifying the location and severity of damage is demonstrated using simulated acceleration data from a three-story steel frame building, and a 15-story building in downtown Los Angeles that is fully instrumented.
\end{abstract}

\section{INTRODUCTION}

Structural health monitoring for civil, mechanical and aerospace systems has been a subject of continuous research over the past few decades. As a result there exists a vast amount of different structural health monitoring (SHM) methods [1]. Physics-based model techniques, in particular, utilize the laws of physics to build the governing equations of motion that describe the behavior of the structure, and are able to identify the location and size of damage [2], as well as predict safety of the structure given the identified structural state. Existing work covers simulated data and linear finite element models [3], real data collected from small-scale steel building laboratory setups $[4,5]$, or from full-scale sliced buildings on shake tables [6,7], full-scale low-rise reinforced concrete buildings [8], as well as nonlinear finite element models using simulated data from multi-story buildings [9].

Department of Mechanical and Civil Engineering, California Institute of Technology, Pasadena, CA, 91125, U.S.A. 
Monitoring of full scale civil infrastructures is still at a preliminary phase, with many of the damage identification techniques being challenging to implement effectively in practice. Limitations of existing SHM techniques stem mainly from modelling assumptions that give rise to inadequate mathematical models failing to describe complex nonlinear behavior. The uncertainties due to such modeling errors are further magnified by the limited number of sensors available and the unavoidable measurement errors. SHM algorithms addressing this ill-conditioned problem often lead to poor performance in terms of inaccurate predictions, missing damage events or initiating false alarms.

Recently, there is an unprecedented increase in the number of real-time measurements produced by permanent, dense (one or more tri-axial sensors per floor) accelerometer arrays in buildings. An example of such arrays is the Community Seismic Network (CSN) [10]. CSN is a Cloud-based strong-motion network, currently comprising hundreds of inexpensive MEM accelerometers located in the greater Los Angeles area. The combination of building-specific high-fidelity non-linear 3D finite element models with such big-data sets provided continuously by the Cloud-based technology has the potential of unlocking new developments in model updating and damage identification techniques for assessment of structural condition during or after strong-motion earthquakes.

In this work, L1 regularization [11] and sparse Bayesian learning tools [12,13] currently developed for linear models of structural behavior and using modal properties, are found to be suitable methods to account for the sparsity of the damage and to reduce ill-conditioning encountered in damage identification techniques. In the present paper we adopt and further extend the above methods to handle model nonlinearities that better reflect damage to the structure. To account for possible model nonlinearities, the damage identification problem is formulated fully in the time domain using response time history measurements. The effectiveness of the methodologies is explored using linear and nonlinear models with simulated measurements and damage scenarios from a 3-story steel frame building and an existing 15-story building in downtown Los Angeles that is instrumented.

\section{DAMAGE IDENTIFICATION UTILIZING RESPONSE TIME HISTORIES}

Let $D=\left\{\hat{\boldsymbol{x}}(k) \in R^{N_{0}}, k=1, \cdots, N_{D}\right\}$ be the measured acceleration time histories, collected from a network of sensors, $N_{0}$ is the number of measured degrees of freedom (DOF), and $N_{D}$ is the number of the sampled data at time instances $t_{k}=k \Delta t$ corresponding to a sampling rate $\Delta t$. Consider also a parameterized class of linear or nonlinear structural models used to model the dynamic behaviour of the structure and let $\theta \in R^{N_{\theta}}$ be the set of free structural model parameters to be identified using the measured response time histories. The parameters of the model are associated with local properties (e.g. stiffness of elements or substructures) of the finite element model. Changes in the values of these parameters are indicative of damage in the structure. The model is parameterized so that the undamaged state corresponds to $\boldsymbol{\theta}=\mathbf{0}$, while damage corresponds to negative values of $\boldsymbol{\theta}$. To cover all possible damage scenarios, the number of parameters may be relatively large compared to the information contained 
in the data. Let $\left\{\boldsymbol{x}(k ; \boldsymbol{\theta}) k=1, \cdots, N_{D}\right\}$ be the model predictions of the acceleration time histories at the measured DOF, obtained for a particular value of $\boldsymbol{\theta}$.

As mentioned above, damage in a structure is expected to be sparse (e.g. confined in a small number of stories in a multi-story building). Thus changes in the values of the parameters are expected to occur only in a few parameters. L1 regularization and sparse Bayesian learning techniques are used to enforce sparse changes in the values of the parameters due to damage.

\section{L1 Regularization Technique}

Regularization is a well-known approach to tackling ill-conditioned inverse problems. L1 regularization specifically is known to enforce sparsity in the solution [14]. Using L1 regularization, the objective function $J_{\text {aug }}(\boldsymbol{\theta})$ to minimize is the measure of mismatch between the measured and model-predicted response time histories, augmented by the L1 regularization term. More specifically:

$$
J_{\text {aug }}(\boldsymbol{\theta})=J(\boldsymbol{\theta})+b\left\|\boldsymbol{\theta}-\boldsymbol{\theta}_{0}\right\|_{1}
$$

where

$$
J(\boldsymbol{\theta})=\sum_{k=1}^{N_{D}} \frac{\|\boldsymbol{x}(k ; \boldsymbol{\theta})-\hat{\boldsymbol{x}}(k)\|_{2}^{2}}{\|\hat{\boldsymbol{x}}(k)\|_{2}^{2}} .
$$

Note that $\boldsymbol{\theta}_{0}$ here is the nominal (undamaged state) parameter values. It is easy to see that the second term of the objective function "pulls" the parameters towards their undamaged state, enforcing sparsity in the damage. A common method to choose the regularization constant $b$ is through the use of the L-curve [15].

\section{Sparse Bayesian Learning Technique}

The sparse Bayesian learning (SBL) framework [12,13] is also used to identify sparse structural damage distributed throughout the structure. For this, prediction errors measuring the discrepancy between the measured and the model-predicted response time histories are modeled by zero-mean Gaussian distributions. The prediction errors of response time histories at different time instants are assumed to be independent Gaussian variables with equal variances $\sigma^{2}$ for all sampling data of a response time history. The prediction errors between different responses are also assumed to be independent. To enforce sparsity, the prior distribution of the $i^{\text {th }}$ model parameter $\theta_{i}$ is parameterized using a zero-mean Gaussian distribution with variance $1 / a_{i}$ and the introduced parameters $a_{i}$ are used as hyperparameters (parameters of the prior distribution).

Bayesian learning is used here to estimate the optimal values of the structural model parameters $\boldsymbol{\theta}$, the prediction error parameter $\sigma^{2}$ and the prior hyperparameters $\alpha=\left[a_{1}, \ldots a_{N_{\theta}}\right]$. The idea is to develop the posterior distribution of the structural model 
parameters given the data, the prediction error parameter and the prior hyperparameters. The selection of the optimal values of the prediction error parameters and the prior hyperparameters is formulated as a model selection problem where the evidence is optimized with respect to prediction error parameters and prior hyperparameters.

Following the sparse Bayesian learning formulation, the optimal value $\hat{\boldsymbol{\theta}}=\hat{\boldsymbol{\theta}}(\boldsymbol{\sigma}, \boldsymbol{\alpha})$ of the model parameter $\boldsymbol{\theta}$ for given $\boldsymbol{\sigma}$ and $\boldsymbol{\alpha}$ correspond to the most probable model maximizing the negative of the log posterior probability distribution function of the model parameters, readily shown to be given by

$$
\bar{J}(\boldsymbol{\theta})=\frac{1}{\sigma^{2}} J(\boldsymbol{\theta})+\sum_{j=1}^{N_{\theta}} \alpha_{j} \theta_{j}^{2}
$$

where $J(\boldsymbol{\theta})$, given by equation (2), is the average measure of the discrepancy between the measurements and the model predictions. It should be noted that the optimal value $\hat{\boldsymbol{\theta}}$ depends on the values of the prediction error parameters $\boldsymbol{\sigma}$ and the prior hyperparameters $\boldsymbol{\alpha}$. Finding the optimal values of these parameters is formulated as a model selection problem. Following Bayesian model selection and using asymptotic approximation to simplify the multidimensional evidence integral, valid for large numbers of data, it can be shown that among all values of the prediction error model parameters $\boldsymbol{\sigma}$ and the prior hyperparameters $\boldsymbol{\alpha}$, the most preferred values $\hat{\alpha}_{j}$ and $\hat{\sigma}^{2}$ are the ones that maximize the evidence of the model, satisfying the following expressions

$$
\begin{gathered}
\hat{\alpha}_{j} \sim \frac{1}{\hat{\theta}_{j}^{2}(\hat{\boldsymbol{\sigma}}, \hat{\boldsymbol{\alpha}})} \\
\hat{\sigma}^{2} \sim J(\hat{\boldsymbol{\theta}}(\hat{\boldsymbol{\sigma}}, \hat{\boldsymbol{\alpha}})) .
\end{gathered}
$$

Note that equations (4) and (5) are nonlinear functions of $\hat{\sigma}^{2}$ and $\hat{\alpha}_{j}$. In order to find a solution, one requires the estimation of $\hat{\boldsymbol{\theta}}(\hat{\boldsymbol{\sigma}}, \hat{\boldsymbol{\alpha}})$ obtained by minimizing equation (3). The structure of equations (3), (4) and (5) can be used to solve this problem in an iterative manner as follows. Select starting values for the prediction error parameters $\hat{\boldsymbol{\sigma}}^{(0)}$ and $\hat{\boldsymbol{\alpha}}^{(0)}$ and estimate $\hat{\boldsymbol{\theta}}^{(1)}=\hat{\boldsymbol{\theta}}\left(\boldsymbol{\sigma}^{(0)}, \boldsymbol{\alpha}^{(0)}\right)$ by minimizing equation (3). Iterate over $k$ until convergence is achieved, by performing the following two steps to update the current values of $\hat{\boldsymbol{\theta}}^{(k-1)}, \boldsymbol{\sigma}^{(k-1)}$ and $\boldsymbol{\alpha}^{(k-1)}$ :

- Minimize equation (3) for estimating $\hat{\boldsymbol{\theta}}^{(k)}=\hat{\boldsymbol{\theta}}\left(\boldsymbol{\sigma}^{(k-1)}, \boldsymbol{\alpha}^{(k-1)}\right)$

- Update the values $\boldsymbol{\sigma}^{(k)}$ and $\boldsymbol{\alpha}^{(k)}$ using equations (4) and (5) with $\hat{\boldsymbol{\theta}}$ replaced by the current $\hat{\boldsymbol{\theta}}^{(k)}$.

The proposed methodology considered here extends sparse Bayesian learning methodologies $[12,13]$ developed for linear finite element models using modal data. Our formulation demonstrated the applicability of the method to linear and nonlinear finite element models based on full response time history measurements. 


\section{APPLICATIONS}

\section{Case 1: Three-Dimensional Single Bay 3-Story Moment Frame Building}

The first structure considered is a three-dimensional single-bay 3-story steel moment frame (Figure 1a). Guided by observed steel-moment frame damage patterns [16], we consider the structure to be damaged due to cracks that have developed in the beam-column connections. Fiber elements are used for the frames, allowing for realistic behavior associated with the opening and closing of cracks to be incorporated into the modeling. Due to the presence of a crack, the local stiffness is reduced when the crack is under tension, while its ability to bear compressive loads is unaltered when the crack closes under compression. To realistically model such stiffness changes due to the fracture of a connection we make use of small-length fiber elements for the beam ends with uniaxial bilinear stress-strain behavior at the lower or upper flanges of the beams. The model parameters are associated with the fiber uniaxial material modulus of elasticity in tension. The modulus of elasticity in compression remains unaltered and equal to the nominal undamaged value. As a result, nonlinearities due to crack opening are expected to be activated during low, moderate and severe earthquake events as well as ambient vibrations.

The finite element model is developed in OpenSEES. The finite element analysis is fully integrated with Matlab where the formulations discussed in the previous section are implemented. The initial values of the model parameters are selected to be the ones corresponding to the undamaged state of the structure.

In the results presented here, the model is parameterized so that one parameter per story is used. In each story the substructures associated with the parameter are the beam ends. Each story parameter accounts for the fractional change from the nominal values of the stiffness in tension of the flange fibers of the beam ends. This fractional change is assumed the same for all beam ends of a story. We simulate data by applying damage to the first story (50\% reduction of the modulus of elasticity of the flange fibers in tension). The parameter values used for the simulated data are $\boldsymbol{\theta}=\left[\begin{array}{lll}-0.5, & 0, & 0\end{array}\right]$. For

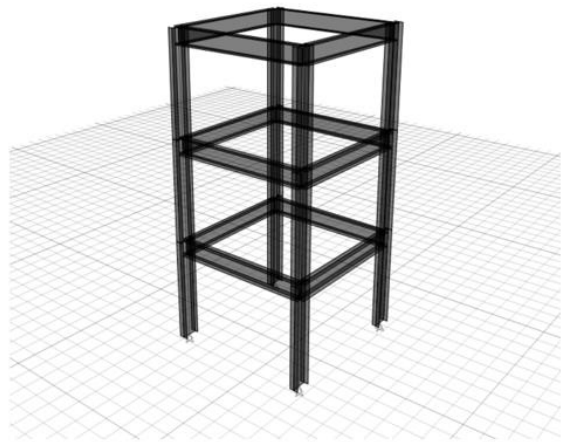

(a)

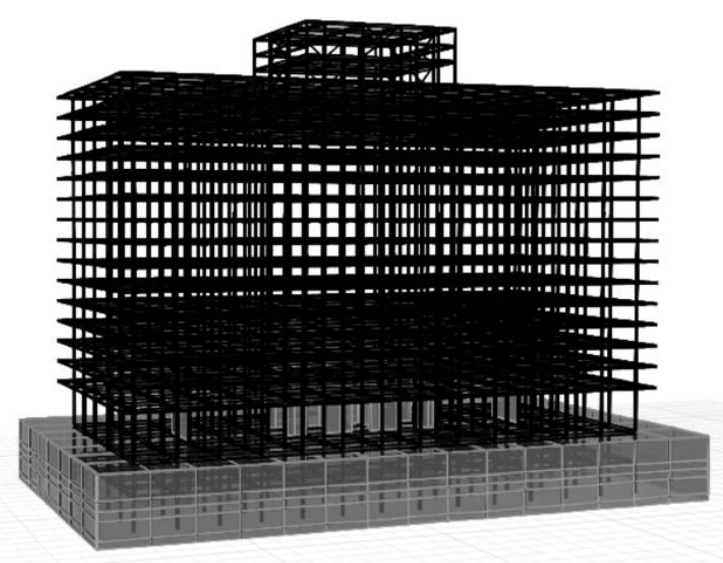

(b)

Figure 1. The two example structures considered. 
TABLE I: CASE 1 (3-STORY BUILDING) RESULTS

\begin{tabular}{|c|c|ccc|}
\hline Data & Method & $\theta_{1}$ (story-1) & $\theta_{2}$ (story-2) & $\theta_{3}$ (story-3) \\
\hline \multirow{3}{*}{ No error } & LSE $(\mathrm{b}=0)$ & -0.4860 & -0.0513 & -0.0555 \\
& L1 $(\mathrm{b}=0.001)$ & -0.4999 & 0.0000 & -0.0001 \\
& SBL & -0.4820 & -0.0001 & 0.0000 \\
\hline \multirow{2}{*}{ Model \& } & LSE $(\mathrm{b}=0)$ & -0.4814 & -0.1532 & -0.1692 \\
Meas. Error & L1 $(\mathrm{b}=0.631)$ & -0.4963 & -0.0001 & 0.0000 \\
& SBL & -0.4979 & 0.0009 & 0.0000 \\
\hline
\end{tabular}

the measured data we assume we have one tri-axial accelerometer per floor. In the analysis we use only the horizontal DOF. Simulated data with and without measurement and modeling error are considered. The simulated data are generated by subjecting the building to a scaled version of an earthquake ground acceleration recorded at the NishiAkashi station during the 1995 Mw6.9 Kobe Japan earthquake. Measurement error is considered by adding zero-mean Gaussian white noise in the simulated time history responses data, with standard deviation $1 \%$ of the response intensity. Model error is considered by perturbing the properties of the nominal model used to simulate the measurements. A zero-mean Gaussian perturbation from the nominal values is assumed with standard deviation $1 \%$ of the nominal values.

Results regarding the parameter values obtained from different methods and cases are presented in Table I. The different approaches listed are normal least squares error which is L1 with the regularization constant fixed to $b=0$ (LSE), L1 regularization with the regularization constant found through the L-curve criterion (L1), and sparse Bayesian learning (SBL). Both SBL and L1 are effective and robust in identifying the location and size of damage under the measurement and modeling errors considered. The SBL technique provides slightly better estimates than the L1 norm technique. Moreover, SBL converges faster, within a few iterations, while the L1 norm technique requires the solution of multiple optimization problems for different $b$ values in order to construct the L-curve. The standard least squares method provides reasonable results, although it spreads out the detected damage to the other stories as well. L1 regularization and the SBL methods are expected to scale better for problems with larger numbers of parameters, where the ill-conditioning and sparsity of damage will be more dominant.

\section{Case 2: Multi-Bay 15-Story Building}

The second structure considered is a real-world, multi-bay, 15-story building located in downtown Los Angeles (Figure 1b). The building is a moment resisting steel frame structure, with concrete shear walls at the basement levels extending up to the second story for drift control. This building is fully instrumented by the Community Seismic Network (CSN) with one to two sensors per floor. The linear finite element model is developed in OpenSEES and is calibrated using measured data from the CSN network.

One stiffness-related parameter per story, for the lowest 4 stories, is used to parameterize the model. The parameter for a story is associated with the stiffness of all beam elements of the story. Damage is simulated by reducing the stiffness of the beams in the first story by $50 \%$. Data is simulated from the nominal model with $50 \%$ reduced 
TABLE II: CASE 2 (15-STORY BUILDING) RESULTS

\begin{tabular}{|c|c|cccc|}
\hline Data & Method & $\theta_{1}($ story-1) & $\theta_{2}$ (story-2) & $\theta_{3}$ (story-3) & $\theta_{4}$ (story-4) \\
\hline \multirow{2}{*}{ No error } & LSE $(\mathrm{b}=0)$ & -0.4588 & -0.0230 & 0.0105 & -0.0096 \\
& SBL & -0.5000 & 0.0000 & 0.0000 & 0.0000 \\
\hline Model \& & LSE $(\mathrm{b}=0)$ & -0.4636 & -0.0264 & 0.0110 & -0.0133 \\
Meas. Error & SBL & -0.4997 & -0.0032 & 0.0000 & -0.0003 \\
\hline
\end{tabular}

stiffness in the first floor. The same excitation time history as in the first example case is applied. Different dataset cases with and without modeling and measurement errors are considered. The identification is done based on low amplitude vibrations so that the structure is in the elastic regime. For the measured data, like before, we assume that we have one tri-axial accelerometer per floor. In the analysis we use only the horizontal DOF.

Results are presented in Table II for the standard least squares error approach (LSE) and the sparse Bayesian learning technique (SBL). The SBL approach provides superior results to the standard LSE approach for the different cases associated with the noise levels. As before, LSE is less effective in reliably estimating the location and size of damage, indicating slight damages in higher floors.

\section{CONCLUSIONS}

L1 regularization and sparse Bayesian learning techniques are developed and tested for their effectiveness for identifying the location and size of damage in buildings. Encouraging results are obtained by applying the methods to selected types of linear and nonlinear models of building and damage scenarios using simulated acceleration response time histories. The effectiveness of the methods is demonstrated to be robust to the modeling and measurement uncertainties considered. Further studies are needed to explore fully the capabilities of the proposed methods in relation to the large number of possible damage scenarios (for example the number of parameters, type of damage mechanisms, modeling fidelity, modeling nonlinearities) and the effect of modeling and measurement error, before one proceeds to validation with real measurements from actual buildings. Due to the large size of the finite element model, the computational effort can be quite significant, as in the case of the 15-story building considered in this study. For high fidelity nonlinear models introduced to model realistic damage scenarios due to cracks developed at the connections between the beam and the columns, the computations may exceed computer requirements. Future work will also concentrate on managing computational effort utilizing model reduction and surrogate techniques. The proposed damage identification methodology, coupled with measurements produced by permanent, dense accelerometer arrays in buildings provided by the Community Seismic Network, is expected to unlock new developments in model updating and damage identification techniques for near real-time assessment of structural condition during or after a strong-motion earthquake. 


\section{REFERENCES}

1. Doebling, S.W., Farrar, C.R. and Prime, M.B., 1998. "A summary review of vibration-based damage identification methods," Shock and vibration digest, 30(2), pp.91-105.

2. Teughels, A., Maeck, J. and De Roeck, G., 2002. "Damage assessment by FE model updating using damage functions," Computers \& structures, 80(25), pp.1869-1879.

3. Johnson, E.A., Lam, H.F., Katafygiotis, L.S. and Beck, J.L., 2004. "Phase I IASC-ASCE structural health monitoring benchmark problem using simulated data," Journal of engineering mechanics, 130(1), pp.3-15.

4. Bernal, D., Dyke, S.J., Lam, H.F. and Beck, J.L., 2002. "Phase II of the ASCE benchmark study on SHM," In Proceedings of the 15th ASCE engineering mechanics conference. Columbia University.

5. Dyke, S.J., Bernal, D., Beck, J.L. and Ventura, C., 2001. "An experimental benchmark problem in structural health monitoring," In Proceedings of the 3rd International Workshop on Structural Health Monitoring (pp. 488-497). CRC Press.

6. Moaveni, B., Conte, J. P. \& Hemez, F. M., 2009. "Uncertainty and sensitivity analysis of damage identification results obtained using finite element model updating," Computer-Aided Civil and Infrastructure Engineering, 24(5), pp. 320-334.

7. Moaveni, B., He, X., Conte, J.P. and Restrepo, J.I., 2010. "Damage identification study of a sevenstory full-scale building slice tested on the UCSD-NEES shake table," Structural Safety, 32(5), pp.347-356.

8. Yousefianmoghadam, S., Behmanesh, I., Stavridis, A., Moaveni, B., Nozari, A. and Sacco, A., 2018. "System identification and modeling of a dynamically tested and gradually damaged 10-story reinforced concrete building," Earthquake Engineering \& Structural Dynamics, 47(1), pp.25-47.

9. Ebrahimian, H., Astroza, R., Conte, J.P. and de Callafon, R.A., 2017. "Nonlinear finite element model updating for damage identification of civil structures using batch Bayesian estimation," Mechanical Systems and Signal Processing, 84, pp.194-222.

10. Clayton, R.W., Heaton, T., Kohler, M., Chandy, M., Guy, R. and Bunn, J., 2015. "Community seismic network: A dense array to sense earthquake strong motion," Seismological Research Letters, 86(5), pp.1354-1363.

11. Hou, R., Xia, Y. and Zhou, X., 2018. "Structural damage detection based on 11 regularization using natural frequencies and mode shapes," Structural Control and Health Monitoring, 25(3), p.e2107.

12. Huang, Y. and Beck, J.L., 2015. "Hierarchical Sparse Bayesian Learning for Structural Health Monitoring with Incomplete Modal Data," International Journal for Uncertainty Quantification, 5(2), pp.139-169.

13. Huang, Y., Beck, J.L. and Li, H., 2017. "Hierarchical sparse Bayesian learning for structural damage detection: Theory, computation and application," Structural Safety, 64, pp.37-53.

14. Bauer, F. and Lukas, M.A., 2011. "Comparing parameter choice methods for regularization of illposed problems," Mathematics and Computers in Simulation, 81(9), pp.1795-1841.

15. Hansen, P.C., 1992. "Analysis of discrete ill-posed problems by means of the L-curve," SIAM review, 34(4), pp.561-580.

16. Miller, D.K., 1998. "Lessons learned from the Northridge earthquake," Engineering Structures, 20(46), pp.249-260. 